\title{
Criminologie
}

\section{Survol bibliographique de la littérature criminologique féminine}

\section{Raymonde Beaudry}

Volume 16, numéro 2, 1983

Les femmes et la justice pénale

URI : https://id.erudit.org/iderudit/017186ar

DOI : https://doi.org/10.7202/017186ar

Aller au sommaire du numéro

Éditeur(s)

Les Presses de l'Université de Montréal

ISSN

0316-0041 (imprimé)

1492-1367 (numérique)

Découvrir la revue

Citer ce document

Beaudry, R. (1983). Survol bibliographique de la littérature criminologique

féminine. Criminologie, 16(2), 121-125. https://doi.org/10.7202/017186ar d'utilisation que vous pouvez consulter en ligne.

https://apropos.erudit.org/fr/usagers/politique-dutilisation/ 


\section{SURVOL BIBLIOGRAPHIQUE DE LA LITTÉRATURE CRIMINOLOGIQUE FÉMININE Raymonde Beaudry*}

Un bilan littéraire des premiers écrits (Lombroso, 1895), jusqu'à nos jours, permet de constater une nette évolution dans la façon $\mathrm{d}$ 'aborder et de traiter le problème de la criminalité chez les femmes.

On sait que les premières études sur ce sujet s'appuyaient sur les statistiques officielles pour en conclure que les délinquantes n'étaient qu'une «minorité marginale», et pour qualifier leur criminalité de «banale». Par la suite, on invoqua les caractéristiques personnelles de la femme, sa nature propre qui en faisait selon les considérations de l'époque, un être inhibé, manipulateur maintenu dans son rôle passif. Ces études reposaient souvent sur des traits physiologiques ou prenaient nettement une connotation psychologique ou psychanalytique. Si l'on se rapporte aux écrits cités dans un article de Groman et Faugeron (1979), on se rend compte qu'il n'y a pas si longtemps, des auteurs comme Pollack (1950), Cowie (1968), Devereux (1970), Vedder et Sommerville (1970), diffusaient encore ces idées sur les femmes délinquantes. Selon Groman et Faugeron, ce n'est que vers les années 75 que ces conceptions ont été sérieusement remises en question (Smart, 1976 ; Bertrand, 1979). Un relevé plus détaillé de tous les travaux est présenté dans le Cahier sur la délinquance des filles, préparé par Biron et al. (1980).

Pour expliquer l'absence des femmes dans les relevés des statistiques of ficielles et afin de parvenir à une vision plus réaliste de la situation, les chercheurs ont développé et perfectionné un instrument de mesure basé sur la délinquance révélée. Les résultats obtenus, suite à l'utilisation de cet instrument, tendent à prouver que la criminalité chez les filles est beaucoup plus remarquable que ce que les statistiques veulent bien laisser croire. C'est ainsi que le débat autour de la nature, de l'importance et de la répartition de la criminalité selon le sexe (Warren, 1981 ; Van Gijseghem, 1980) se poursuit, malgré la prolifération des recherches déjà publiées sur ce sujet. Comme le souligne Canter (1982), la polémique persiste, à savoir laquelle des données officielles ou des mesures de délinquance révélées est la plus adéquate. Cette préoccupation face à l'expansion de la

* Documentaliste, Université de Montréal. 
criminalité chez les femmes se reflète dans le texte de Adler (1981). En s'appuyant sur les données des Nations unies, Adler affirme que la criminalité des femmes dans les pays développés augmente plus vite que la criminalité des hommes.

De même le mouvement d'émancipation de la femme amorcé dans les années 60 et les bouleversements qu'il a suscités, demeurent encore très actuels dans la littérature criminologique. Ce mouvement de libération a servi de prétexte pour tenter d'expliquer la hausse du taux de la criminalité chez les femmes, ou plus particulièrement l'effet du changement des rôles sociaux de la femme sur l'augmentation de ces mêmes taux. Les données obtenues par Steffensmeier (1980) et Austin (1982) réfutent la première hypothèse; pour James et Thornton (1980), le féminisme a eu peu d'effet comme tel sur l'augmentation de la délinquance des filles mais plutôt, serait la cause d'une plus grande agressivité observée chez les délinquantes. Fox (1979) accorde au changement des rôles sociaux de la femme dans la société canadienne une incidence directe sur le taux de criminalité de celle-ci, tandis que Gibbons (1981) n'y voit aucune corrélation. Comme on peut le constater, la controverse subsiste toujours à ce sujet.

Éventuellement, selon Datesman et Scarpitti (1980), les opportunités sociales et économiques offertes à la femme seront des facteurs beaucoup plus déterminants de la criminalité, que ne pourrait l'être la question de l'égalité ou l'inégalité des sexes. En effet, derrière ce prétexte du MLF, les données économiques (Zietz, 1981 ; Chapman, 1980), géographiques (Adler, 1981 ; Conseil de l'Europe, 1980), et historiques (Jones, 1980) sont, semble-t-il, des composantes plus appréciables pour la compréhension du phénomène.

D'autre part, les publications récentes sur la délinquance des filles laissent beaucoup plus la parole aux jeunes, à l'expression de leur vécu, qu'on se réfère ici aux écrits de Gagnon et Langelier-Biron (1982), de B. Koeppel (1980) et à ceux du CNRS (1980). Le milieu psychosocial dans lequel ces jeunes évoluent et les rapports qu'ils entretiennent avec ce milieu sont aussi examinés plus en profondeur, Biron et al. (1980). Afin de mieux comprendre l'expression de l'agressivité chez la fille, et le pourquoi de son comportement violent, il devenait nécessaire d'adapter des instruments de mesure et des échelles de classification proprement spécifiques aux filles. C'est dans ce sens que l'étude de Lacombe et al. (1981), entre autres, innove dans le domaine des études criminologiques. Le livre récent de Campbell (1981) qui aborde la question des bandes de filles apporte également une information nouvelle à ce chapitre. 
Le système de justice pénale et la critique formulée à son égard en ce qui à trait au "paternalisme des juges», à l'inégalité du traitement et des jugements discriminatoires rendus par les tribunaux, ainsi que la grande institutionnalisation qui en résultent, sont des problèmes continuellement soulevés dans la littérature criminologique. Ces principaux thèmes sont analysés dans les publications de certains auteurs comme Bowker (1981), Feinman (1980), Rafter et Stanko (1982), Datesman et Scarpitti (1980), et Smith (1980).

D'autres auteurs comme Henriquez (1982), Stanton (1980), Freedman (1981) et Figuera-McDonough et al. (1981), ont publié des études soit historiques ou descriptives sur les femmes en prison et leurs conditions de vie. Plus spécifiquement, McNulty (1980) et Ann Jones (1980) se sont attardés à étudier la typologie des femmes meurtrières. Ann Jones, après un bref historique de la question, conclut que contrairement à la croyance populaire, il n'y a aucune tendance vers l'augmentation de l'homicide chez la femme et la femme meurtrière n'est pas traitée avec plus de clémence que les hommes. De fait, la plupart des femmes ont des sentences plus longues que celles des hommes pour un même crime. Les monographies de Ford (1981) et Léonard (1981) présentent à cet égard une vision plus globale de la femme criminelle par rapport à la société contemporaine.

Afin de favoriser les échanges interdisciplinaires et d'unir les efforts des recherches dites académiques avec ceux des praticiens, des conférences ayant pour thème "la femme délinquante» (Female Offenders, 1980) ou "la femme et le crime» (Women and crime, 1981) ont aussi fait l'objet de publication.

Pour conclure ce relevé de littérature criminologique, on peut établir que l'approche future susceptible de mieux analyser les problèmes de la criminalité des femmes sera celle qui se voudra multidisciplinaire. En fait, le bilan des dernières publications laissent croire que cette orientation est déjà amorcée.

\section{BIBLIOGRAPHIE}

ADLER, Freda (1981). The Incidence of Female Criminality in the Contemporary world. New York : New York University Press. 275 p.

AUSTIN, R.L. (1982). «Women's Liberation and Increase in Minor, Major, and Occupational Offenses». Criminology 20 (3-4) : 407-430.

BERTRAND, M.A. (1979). La Femme et le crime. Montréal, l'Aurore, 1979. 
BIRON, L.; R. GAGNON, M. LEBLANC (1980). La délinquance des filles. Montréal, Université de Montréal. Groupe de recherche sur l'inadaption juvénile.

BOWKER, L.H. (1981). Women and Crime in America. New York; Macmillan.

CAMPBELL, L.H. (1981). Girl Delinquents. Oxford: Basic Blackwell.

CANTER, Rachelle J. (1982). "Sex differences in self-report delinquency», Criminology 20 3-4 : (373-393).

CENTRE NATIONAL DE LA RECHERCHE SCIENTIFIQUE (1980). Cahiers sur la femme et la criminalité. Paris : CNRS. $192 \mathrm{p}$.

CHAPMAN, J.R. (1980). Economic Realities and the Female Offender. Lexington: Lexington Book. $234 \mathrm{p}$.

CONSEIL DE L'EUROPE (1980). La criminalité des femmes en RFA, en Italie, et en Angleterre et aux Pays de Galles. Strasbourg : CE, $227 \mathrm{p}$.

COWIE, J. et al. (1968). Delinquency in Girls. London : Heinemann. 220 p.

DATESMAN, S.K.; Fr. SCARPITTI, (eds.) (1980). Women, Crime and Justice. Oxford : Oxford University Press. 376 p.

DEVEREUX, G. (1970). Essais d'ethno-psychiatrie générale. Paris : Gallimard. $394 \mathrm{p}$.

FEINMAN, C. (1980). Women in the Criminal Justice System. New York : Praeger. $121 \mathrm{p}$.

FEMALE OFFENDERS (1980). Selected Papers from an International Symposium, Vancouver, Canada, 29 janvier, février 1979. Vancouver : Simon Fraser University Criminology Research Center. 331 p.

FIGUERA-McDONOUGH, J. et al. (1981). Females in Prison in Michigan, 19681978: A Study of Commitment Patterns. University of Michigan, School of Social Work. $162 \mathrm{p}$.

FORD, Lee (ed.) (1981). Women of the Eighties. Vol. 7. The Woman Criminal. Auburn : Ford Assoc. 100 p.

FOX, J.; T.F. HARTRIEGEL (1979). «Changing Social Role and Female Crime in Canada ; a Time Series Analysis», Canadian Review of Sociology and Anthropology 16 (1) : 96-105, 1979.

FREEDMAN, E.B. (1981). Their Sisters'Keepers ; Women's Prison Reform in America, 1830-1930, University of Michigan Press. 248 p.

GAGNON, R., L. LANGELIER-BIRON (1982). Les filles en marge: paroles et réflexions. Université de Montréal, Groupe de recherche sur l'inadaptation juvénile.

GIBBONS, D.C. (1981). Delinquent Behavior. Englewood Cliff, NJ : Prentice-Hall.

GROMAN, D., Cl. FAUGERON (1979). «La criminalité féminine libérée : de quoi?». Déviance et société 3 (4) : 363-376.

HENRIQUEZ, Z.W. (1982). Imprisoned Mothers and their Children: A Descriptive and Analytical Study. Texas : University Press. $226 \mathrm{p}$.

JAMES, J., W. THORNTON (1980). «Women's liberation and the female delinquent", Journal of Research in Crime and Delinquency 17 (2) : 230-244.

JONES, Ann (1980). Women who kill. New York : Holt, Rinehart and Winston. $408 \mathrm{p}$.

KOEPPEL, B. (1980). On m'a placé un peu partout, Vaucresson : Centre de formation et de recherche de l'éducation surveillée. $274 \mathrm{p}$. 
LACOMBE, P., L. BIRON, M. FRÉCHETTE (1981). Instruments de mesure de l'agression et de la violence chez l'adolescente, Montréal : Université de Montréal, G.R.I.J., 230 p.

LÉONARD, E.B. (1981). Women, Crime and Society : A Critique of Theoretical Criminology. New York : Longman, $256 \mathrm{p}$.

LOMBROSO, C., G. FERRERO (1895). The Female Offender. London : Fisher Univen. $313 \mathrm{p}$.

McNULTY, Faith (1980). The Burning Bed : The True Story of a Wife who Killed. New York : Harcourt Brace Jovanovich, $320 \mathrm{p}$.

POLLACK, D. (1950). The Criminality of Women. Philadelphia : University of Pennsylvania Press.

RAFTER, N.H., E.A. STANKO, (eds.) (1982). Judge, Lawyer, Victim Thief: Women, Gender Roles, and criminal justice. Boston : Northeastern University. 383 p.

SMART, C. (1976). Women, Crime and Criminology - a Feminist Critique. London : Routledge \& Kegan, $208 \mathrm{p}$.

SMITH, D.D. (1980). Young Female Offenders: Analysis of Differential Handling based on Sex. Pittsburgh : HCJJ. $12 \mathrm{p}$.

STANTON, A.M. (1980). When Mothers Go to Jail. Lexington : Lexington Book. $224 \mathrm{p}$.

STEFFENSMEIER, D.J. (1980). «Assessing the Impact of Women's Liberation on Sex Based difference in the Handling of Adult Criminal Defendants", Crime and Delinquency 3: 344-357.

VAN GIJSEGHEM, H. (1980), «Le crime féminin et masculin : deux expressions d'une même délinquance", Revue québécoise de psychologie I (1) : 109-123.

VEDDER, C., D. SOMMERVILLE (1970). The Delinquent Girl. Springfield, Ill. : C.C. Thomas. $166 \mathrm{p}$.

WARREN, M.Q. (ed.) (1981), Comparing Male and Female Offenders. Beverly Hills, Calif. : Sage. 144 p.

WOMEN AND CRIME (1981). Papers presented to the Cropwood Round Table Conference, Dec. 1980. Cambridge : University of Cambridge, Institute of Criminology, $141 \mathrm{p}$.

ZIETZ, D.W. (1981). Women who embezzle or Defraud: A Study of Convicted Felons. New York : Praeger. $157 \mathrm{p}$. 